not come to Mahomet, then Mahomet will go to the mountain.

In concluding, I would ask the Tri-State Society to renew its allegiance to our Code, and press forward to the mark and prize of our high calling. The Society has just cause to be proud of the record that is being made by its members in scientific investigations, and whilst the large part of the glory belongs to those who are investigating and reporting upon the hitherto obscure causes of some of our most common maladies, yet we as a Society can shine in their reflected light. But I have occupied enough of your time, and hence, close, thanking you for having had the privilege and honor of serving you one year as your president.

\section{LYMPHOSARCOMA OF THE MESENTERY.}

(From the Laboratory of Pathology of the Chicago Policlinic.) BY MAXIMILIAN HERZOG, M.D.

PROFESSOR OF PATHOLOGY IN THE CHICAGO POLICLINIC. chICAgo.

Sometime ago Dr. M. L. Harris ${ }^{1}$ and myself re. ported a case of lymphosarcoma of the mesentery operated on successfully in a boy 5 years old. When I then collected the literature of the subject, I was able to tabulate a total of 57 cases of solid mesenteric tumors, of which about 10 were sarcomata. To the latter class I am able to add a new case. As in most instances of solid mesenteric tumors, the exact diagnosis as to location was not made intra-vitam. For a short abstract of the history of the case, I am indebted to Dr. R. B. Preble, under whose care the patient was during his last weeks of life. The history is as follows:

Sept. 14, 1898. H. G., German, 62 years old, physician. He traveled extensively as a young man, living for a time in Asia where he escaped all diseases except a mild malaria; has lived many years in Chicago. A bout six years ago he discovered a moderate glycosuria, which disappeared on a course of Carlsbad treatment. The glycosuria never, to his knowledge, returned. He has been a moderate drinker all his life; never had syphilis. The present illness is of rather indefinite onset both as to time and symptome, but he must have been ail ing for about two months. He has continued to do his medical work up to one week ago, and complains only of weakness, dyspnea, moderate constipation, and of the increasing size of the abdomen. A short time ago he had what he describes as an epididymitis ${ }^{2}$ followed by swelling of the lymph glands be low Poupart's ligament. It continued only a short time, and has now entirely disappeared. He has had no vomiting, no diarrhea, no jaundice, no hemorrhages.

Examination.-Well nourished and rather fat man, appa rently about 60 years old ; no jaundice, no cyanosis; moderate dyspnea, moderate edema of the feet. Lungs: lower bordere high, but move freely on respiration; no rales, no fluid in pleural cavities. Heart : displaced upward by the high dia phragm, but normal in size. Tones pure and of normal inten sity. Pulse: regular, strong, normal. Abdomen : prominent, contains large amount of free fluid, which changes promptly with change of position. No varicose veins. Liver: can no be palpitated, nor outlined by percussion. Spleen: palpable Lymphatic glands : below Poupart's ligament both sides abou the size of a hen's egg; elsewhere not palpable. Urine: contains no sugar, albumin nor casts. Blood examination, negative.

Diagnosis.-A trophic cirrhosis of the liver, based on ascites,

1 Harris and Herzog: Solid Tumors of the Mesentery, etc. Annals of Surgery, July, 1897

With reference to the trouble in the testicles, Dr. Karl Doepfner, who saw the patient during the latter part of August or the first of Sep tember, informs me that there was then noticeable in the head of the left Whether this swelling was a tumor metastasis is rather difficult to say. since it apparently disappeared again. There was never any complaint in the part of the dientieared again. There was never any complain Preble saw him Since I knew nothing about the sympto the the testicle at the time I made the postmortem and sine a partials to tion only was permitted, the testicles were not removed or examined. palpable spleen, absence of any other manifest cause for ascites and the history. The enlarged glands in the groins are not accounted for except as the probable result of an ulcer of the leg.

Treatment.-Diuretics, salines and mild diet.

The fluid in the abdomen gradually increased, causing dyepnea, until it was decided to tap the patient. Withdrew 6000 c.c. of milky fluid, s.g. 1012, large amount of albumin, sugar, 2 per cent. The fluid cleared on being shaken with ether contained fow round mononuclear cells with fat granules. The fluid was that of a chylous and not of an adipose ascites. After withdrawal of the fluid, large numbers of tumor masses could be felt throughout the abdomen. After the tapping, the patient rapidly lost strength, developed an edema of the dependent portions of the body, and on the last day a very slight icterus.

Clinical diagnosis.-Tumor abdominis, probably carcinoma, but there is no evidence pointing to the primary organ. Chylous ascites due to obstruction of thoracic duct by the tumor.

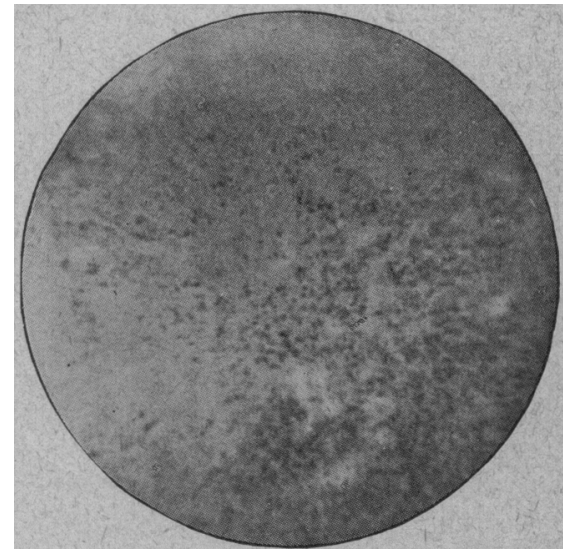

Fig. 1.-Section from tumor mass of the mesentery showing necrobi

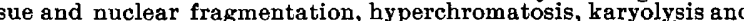
free chromatin granules. Spencer prof. obj. $1 / 4$; oc. 11/2.

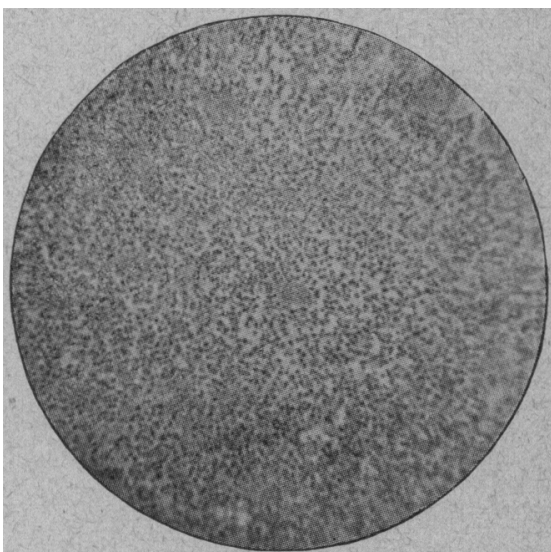

Fig. 2. - Section from tumor mass in inguinal glands showing small blood-vessels and young proliferating cells. Spencer prof. obj. 1/4; oc. $1 \frac{1}{2}$

The postmortem was made by the author, Oct. 16 , 1898-fifteen hours after death. The protocol of the autopsy reads as follows:

The body is that of a very strong, heavy man, about 60 years of age; estimated weight 250 to 260 pounds; well nourished. Postmortem rigidity and lividity well marked; extensive edema of the lower extremities, more marked on the right than on the left side; edema of the scrotum. The skin of the legs is hard and brownish in color. Between the umbilicus and the symphysis pubis, to the left of the linea alba, is a circular trochar wound, almost closed. On making the primary long incision the whole skin over thorax and abdomen is found to be edematous, a clear fluid oozing out from it.

The abdominal cavity contains from two to three gallons of a yellowish, very turbid ascitic fluid. The right pleural cavity contains quite a large amount of 
clear fluid; the exact amount can not be estimated, since on manipulation ascitic fluid from the abdomen runs over into the pleural cavity and mixes with the clear pleuritic transudate. The pericardium is amount of pericardial fluid. The external surface of the heart-which latter is of normal size--shows a moderate amount of fat; the myocardium is soft, flabby and pale; the orifices are normal in size; the endocardium is smooth over its whole extent; the valves are normal. The intima of the large vessels is smooth; the walls of the coronary arteries are somewhat stiff and thickened, their intima, however, is perfectly smooth.

Lungs: No adhesions on either side; quite an amount of clear fluid on the right side. The lower lobe of the right lung shows, on its anterior surface, several subpleuritic, flat, grayish-white patches, raised slightly above the surface; there were also found in the pulmonary parenchyma several firm, hard nodules, likewise of a grayish-white color. The entire lower lobe of the right lung, on section, appears more or less collapsed and solid; pieces from this part, however, still float on water. The lower lobe of the left lung also contains several hard nodules of the same type as those mentioned above. Both lungs are markedly edematous throughout. On the left pleura costalis, about the region of the fifth rib, in the axillary line, are found some small grayish-white, slightly prominent patches. The bronchial glands on either side are enlarged, firm and nodular, and on section, appear grayish-white and somewhat translucent. In the glands, from which small amounts of whitish material may be scraped, are numerous hemorrhagic spots. The omentum majus is studded throughout its whole extent with firm, grayish-white nodules from a few millimeters to one centimeter in diameter.

The spleen is large, smooth, quite firm and darkbrownish-red on section; the trabeculæ stand out distinctly and prominently. The kidneys are of normal size, capsules smooth, easily detachable; cortex and medulla normal in size and proportion; cortical markings fairly distinct. The mucous membrane of the pelvis is smooth. The adrenals, more or less embedded in tumor masses, show very marked pigmentation on section; their substance, however, is uniformly soft. The mesentery, the head of the pancreas, the retroperitoneal glands and the omentum minus form one large, irregular tumor, the component parts of which are united to each other more or less firmly by nodular masses. The mesentery and the retroperitoneal glands form a tumor mass as big as two large fists; the omentum minus is irregularly infiltrated with firm nodules; the head of the pancreas presents a roundish mass four or five centimeters in diameter. In the rest of the pancreas, the glandular tissue appears normal, with the exception of some smal hemorrhagic foci. To the tumor, involving mesentery and head of the pancreas, part of the circumference of the duodenum is firmly attached. The lumen of this gut, however, does not show any marked con striction, and there is no impediment to the flow of the bile, which, on slight pressure upon the gall-bladder, escapes through the common duct. The stomach appears normal. On the under surface of the head of the gall-bladder there are three flat, grayish-white, somewhat prominent subperitoneal patches. The gall-bladder contains thickened, cloudy, dark-green viscid bile. Its mucous membrane is smooth.
The liver is small in size, nodular and uneven over its entire surface. The liver tissue is firm and uneven on section. Masses from the size of a pin-head to that of a millet seed rise above the level of the cut surface and make it uneven. Several small nodular, grayish-white, enlarged lymph-glands are found behind and around the thoracic anrta directly above the diaphragm. The lymphatic vessels can be followed downward behind the peritoneum, as several parallel white bands extending far down into the pelvis. The left inguinal glands are hard and firm, enlarged to the size of a large walnut, and on section, are found to be studded with grayish-white translucent nodules.

Anatomic diagnosis.-Malignant neoplasm of the mesentery and retroperitoneal glands; metastases in the head of the pancreas, the omentum minus and majus, the lymphatic glands on the neck of the gallbladder, the pleura costalis and pulmonalis, the bronchial glands on either side the lymphatics along the thoracic and abdominal aorta, and the left inguinal glands. Compression atelectasis of the lower lobe of the right lung. Chronic passive congestion of the spleen. Cirrhosis of the liver. General anasarca.

Microscopic examination.-The tumor masses in the mesentery, in the omentum, in the head of the pancreas, in the inguinal glands, and in the lungs, are all of the same structure. They consist of small, round cells of the type of the ordinary small lymphoid cell. The nucleus is quite rich in finely glanular chromation, the cell protoplasm very scanty. Besides those cells there are found in other areas round cells, slightly larger than the former; they are more of the type of those cells found in the proliferating center of Flemming in the lymph follicle. Their nuclei are larger, and the chromatin is not as finely, but more coarsely granular; the cell protoplasm is not quite as scanty. The tumor cells are contained in a meshwork or matrix of fine connective tissue fusiform cells and fibers. These cells and fibers are not very abundant, still they are much better developed than they are in the normal lymph follicle. The blood-vessels in the tumor masses are moderately numerous and not very large. In places free blood is found between the tumor cells. Large areas of the neoplastic tissue show necrobiotic changes. All cells in such territories have lost their nuclei, and the cell body has become somewhat swollen and indistinct in outline; the degeneration has, however, nowhere progressed to the formation of hyalin or colloid material. This is noted expressly, since in the other case of lymphosarcoma of the mesentery reported by Dr. M. L. Harris and myself ${ }^{3}$ there was very extensive colloid degeneration.

The above microscopic description refers more or less to all the tumor masses. However, it ought to be stated that the most marked and most extensive regressive changes are present in the large tumor mass, found in and developed from the mesentery. Here we see great tracts of necrobiotic tissue and also areas of fatty degeneration. The areolar tissue itself presents fatty changes; its meshwork is very poor in nuclei and shows a great deal of fine vacuolation. The necrobiotic areas, in a finely granular mass, show comparatively fow nuclei with hyperchromatosis, nuclear fragmentation, karyolysis and free chromatin granules. The tumor mass most distant from the mesenteric tumor, namely, the one in the inguinal glends, on the other hand, shows very few regressive changes, 
but presents clear and distinct cells and like nuclei, some with karyokinetic figures. Evidences of fat necrosis were not found.

The body of the pancreas is fairly normal, so far as the parenchymatous glandular tissue is concerned; the interstitial connective tissue, especially that around the small ducts, is increased in amount.

In the liver there is seen a proliferation of the intra-acinous periportal connective tissue; there is, however, no proliferation of the intra-acinous connective tissue. The proliferative processes are evidently active and progressive, since the intra-acinous tissue shows numerous newly formed blood-vessels and a proliferation of the bile-ducts. The tissue between the lobules also shows a very abundant infiltration, with small, round cells, all of the type of small lymphoid cells. Polymorphonuclear leucocytes were not found in the intra-acinous septa. The liver-cells proper are normal in size and arrangement. There is very little fatty degeneration in the lobules. Many of the intralobular bile capillaries, especially those near the periphery of the lobule, are much distended and filled with bile pigment. The liver-cells in the immediate neighborhood of such distended bile capillaries contain a considerable amount of granular bile pigment.

Epicrisis.-The mesentery, in which was found by far the largest tumor mass, was undoubtedly the pri. mary seat of the lymphosarcoma, which subsequently gave rise to metastases in the pancreas, omentum, lungs, a number of lymphatic glands, etc.

The ascites, as such, was due to the cirrhosis of the liver. Its peculiar nature, i. $\theta$., that of a chylous ascites, must be explained by pressure on, and perhaps complete obstruction of, the thoracic duct, by lymph-glands enlarged and hardened by tumor metastases. The sugar found in the peritoneal fluid may be looked upon as a vicarious excretion of a pathologic metabolic product due to the extensive pancreatic involvement.

It is by no means obvious that the advanced cirrhosis of the liver in the present case was due entirely or even partly to alcohol. The deceased had certainly during the last six years of his life, i. e., after his transitory diabetic attack, been a very moderate drinker only, and he had never had any symptoms of cirrhosis except during the last weeks of his life. It does not appear entirely unreasonable to assume that the advanced cirrhosis of the liver, giving rise to definite symptoms only toward the later part of a comparatively short illness, may have been due to toxins derived from the large and widely spread sarcomatous masses in the abdominal cavity and brought to the liver by the portal circulation.

This possibility, I think, can not be viewed as an absurdity, but rather deserves some consideration, if we look upon it in the light of the contributions upon the subject of cirrhosis of the liver by Adami ${ }^{4}$ and by Bektoen and Weaver, who quite recently reported to the Chicago Pathological Society observations on cirrhosis of the liver in the guinea-pig, due to bacterial infection and to the circulation of toxins in the liver.

+ Adami : Montreal Med. Journal, September and December, 1898.

For Vulvar Pruritis.-Corrosive sublimate, 40 centigrams; alum, 8 grams; water, 1 licer; methylene blue, q. s. External application.-Guinard in Progres Méd., January 7.

\section{THE VARIABILITY OF DISEASE GERMS}

BY J. F. PEAVY, M.D.

\author{
ASHEVIYLE, N. C.
}

The theory of descent with modifications produced through the action of natural causes has been accepted by the best scientific minds of the age as expressing nature's method in the elaboration of organic forms. In no other way, perhaps, has this doctrine been of such signal service to science in general as through its influence upon the methods of studying and interpreting natural phenomena. Especially in the biologic sciences has this principle of development, of progressive modification, furnished the key for the solution of numerous problems. In the light of this principle, differences of kind are seen to arise through the accumulation of differences of degree, and, further, that these organic modifications arise through adaptation to the condition of life. The life of an organism is, in fact, a series of adjustments to varying conditions and forces which affect it.

Hitherto, our observations and generalizations have related chiefly to the higher organic forms. The unity of nature demands that we extend the general principles of biology to the lowly organized forms as well, among them the micro-organisms which are the specific causes of disease. The low forms are more distinctly the products of their immediate physical environment than the higher ones, and hence more directly affected by variations in it. The higher forms have more of accumulated property or momentum, of established biologic habit, which enables them to resist more effectively the influence of changes in physical environment.

The physical conditions with which the vital activ. ities of micro-organisms are correlated relate especially to moisture, temperature and food-supply. Light, oxygen-supply, electric conditions and the presence of competing organisms exert important modifying influences in certain instances. Variations in physical environment tend to produce responsive variations in the activities of the organisms them. selves, which relate especially to nutritive and repro. ductive activity.

On the question of morphologic variation, practical working bacteriologists are slow to admit the truth. Indeed, the attitude of many of them is a fine illus tration of the strength of preconceived opinion. Wed. ded to the notion of the immutability of species in this department of biology, believing that each type is distinct and unvarying, they are blind to the real significance of facts which they will perhaps in a general way admit. The ordinary methods for the laboratory cultivation of bacteria are, in fact, not calculated to allow of variation. Everything is usually done by exact methods; the culture-media made by precise formulæ; incubation carried on at uniform tempera. tures; the environment, in fact, kept as unvarying as possible. With absolutely uniform environment, organic variation could nover occur, except such as pertains to the life cycle of each individual. Under natural conditions no such uniformity exists, . but variety is the rule, and thus arises the opportunity, indeed the necessity, for organic variation. Notwithstanding the unfavorable conditions, many bacteriologists are beginning to admit the principle of variability as applied to disease germs. Under the name of "biologic modifications," Sternberg not only admits the principle but lays considerable stress upon it. 